\title{
EEG Feature of Guess the coin game of human-computer
}

\author{
Wang ping, Mu Zhendong ${ }^{1}$ \\ College of Information Engineering, Jiangxi College of Technology, Jiangxi Nanchang 330098
}

Keywords: Human-computer decision; Guess the coin game; EEG

\begin{abstract}
The decision is the selection process in order to achieve a certain purpose of different decision schemes, whether can reflect the different features from the EEG, is an objective study of the decision making process based on EEG, this paper use man machine game mode, choose the game mode of telecom research guess coin signal characteristics of brain, by designing the different guess coin mode induce EEG, ERP analysis of chemical composition of these EEG signal, analysis of subjects of different EEG features guess coin mode.
\end{abstract}

\section{Introduction}

The decision is in order to achieve a certain purpose, adopt what strategy and method, choose the one that best achieve their satisfaction judgment process scheme from alternative solutions, research on decision-making process, once is an economist and mathematician in the world, when the brain imaging technology, the study of neural psychological mechanism of decision-making behavior in is not a a "black box", nowadays many researchers began to study on the neural mechanism of decision making process with brain imaging techniques, thus revealing the decision-making process operation process of human brain.

Research on decision-making process, at first in the monkey as the research object, Platt and Glimcher in 2003, was found in the monkey stone / hammer / cloth in the game, the lateral intraparietal area (lateral intraparietal area, LIP) neurons coding may benefit behavior, also is the decision-making process of the expected relative performance. In the selection, there are obviously difference between animal and human, many researchers in recent years to study the neural mechanisms of human decision making. Paulus in 2004 for children of hammer / scissors / cloth Game Research of eeg. Minati in 2012 by fMRI and EEG as the tool to analyze the economic behavior of people in the profit and loss, through the analysis of the frequency of EEG, studied the effects of different economic parameters of different brain regions produce; Ruz in 2012 by two people cooperation, to emotional volatility to study the peer trust process, functional analysis of different brain region as a tool using ERP; Billeke in 2012 through the study of the social process to study the brain on the risk expected response, through the research on ultimatum decision modeling, analysis of different EEG frequency band response, through the study of different cerebral regions related to the medial prefrontal, display and social risk sensitivity related EEG features.

This paper choose guess coin game as the experimental object, the experimental object is human and computer, to detect EEG of different patterns, analysis the difference between different modes of brainwave components, and using the difference between these EEG components, extracting their

$\overline{1 * \text { Corresponding Author: } 418623577 @ \text { qq.com }}$ 
features using Fisher distance, the results show that the characteristics of EEG of people produced in but the selection process with a certain psychological game components.

\section{The experimental process}

In this paper, the use of EEG in brain computer interface Laboratory of Jiangxi Institute of technology acquisition, using the device is a Neruoscan40 channel EEG acquisition instrument, the software used is Scan4.3, the experimenter sat in a shielded room, put a screen positive stimulus, shown above to be carried out by the stimulus program, experimental process, subjects according to the laboratory experiment guidance.

In the experiment, the computer has a coin, experimenters have a coin, when subjects and computer coins when choosing the same face, the experimenter losing a point, on the other hand, computer losing a point, the initial points are 25 points, each game cycle is divided into the following stages:

1, the preparatory period: display the countdown, reminding participants prepare to choose surface;

2, beginning of period: computer display selected face of a coin;

3 , period of reflection: according to the selection results, deducted from the related points, the experimenter to prepare for the next game cycle;

The preparatory period, the countdown total time $1200 \mathrm{~ms}$, beginning of period $1000 \mathrm{~ms}$ period of time consuming, time-consuming $5000 \mathrm{~ms}$, the whole experiment period time consuming $7200 \mathrm{~ms}$, when one side scores 0 or experimental time up to 15 minutes time, the end of an experiment, the subjects were resting, prepare for the next experiment.

The experiment adopts the method of reference electrode as reference electrode on the right mastoid process, using $1000 \mathrm{~Hz}$ sampling rate, band acquisition using $200 \mathrm{~Hz}$ low pass, high pass $0.05 \mathrm{~Hz}$ and $50 \mathrm{~Hz}$ trap.

\section{The results of ERP}

When the experimental design, this paper responses for subjects expectations can be described as the following process: the first cycle computer random coins, subjects because no reference, so also random coins, this cycle may appear in four ways (computer and subjects of coins respectively: positive - negative, positive, anti - positive, anti - anti), the experiment was divided into two ways, one is before the experiment, the experimental officer not to give the subjects any reminders, another is before the experiment, laboratory technician told the subjects computer next coin $50 \%$ and the same; when two consecutive occurrences of the same plane, 60 opposite computer next time, if 3 consecutive identical, then the $80 \%$ anti, followed by calculation. When the first cycle is complete, thinking period subjects will think according to the laboratory technician's guidance, the next should be what is appropriate. So the game brain waves will appear in the $2200 \mathrm{~ms}-5000 \mathrm{~ms}$, after removing the visual impact time, we chose the $1300 \mathrm{~ms}$ time $(2700 \mathrm{~ms}-4000 \mathrm{~ms})$ as the analysis object, under the 10-20 standard, we select four electrodes as the analysis object (FP1, F8, FC3, and T3), as shown in Figure 1 EEG signals, can be clearly seen from the difference between the two experimental subjects. 

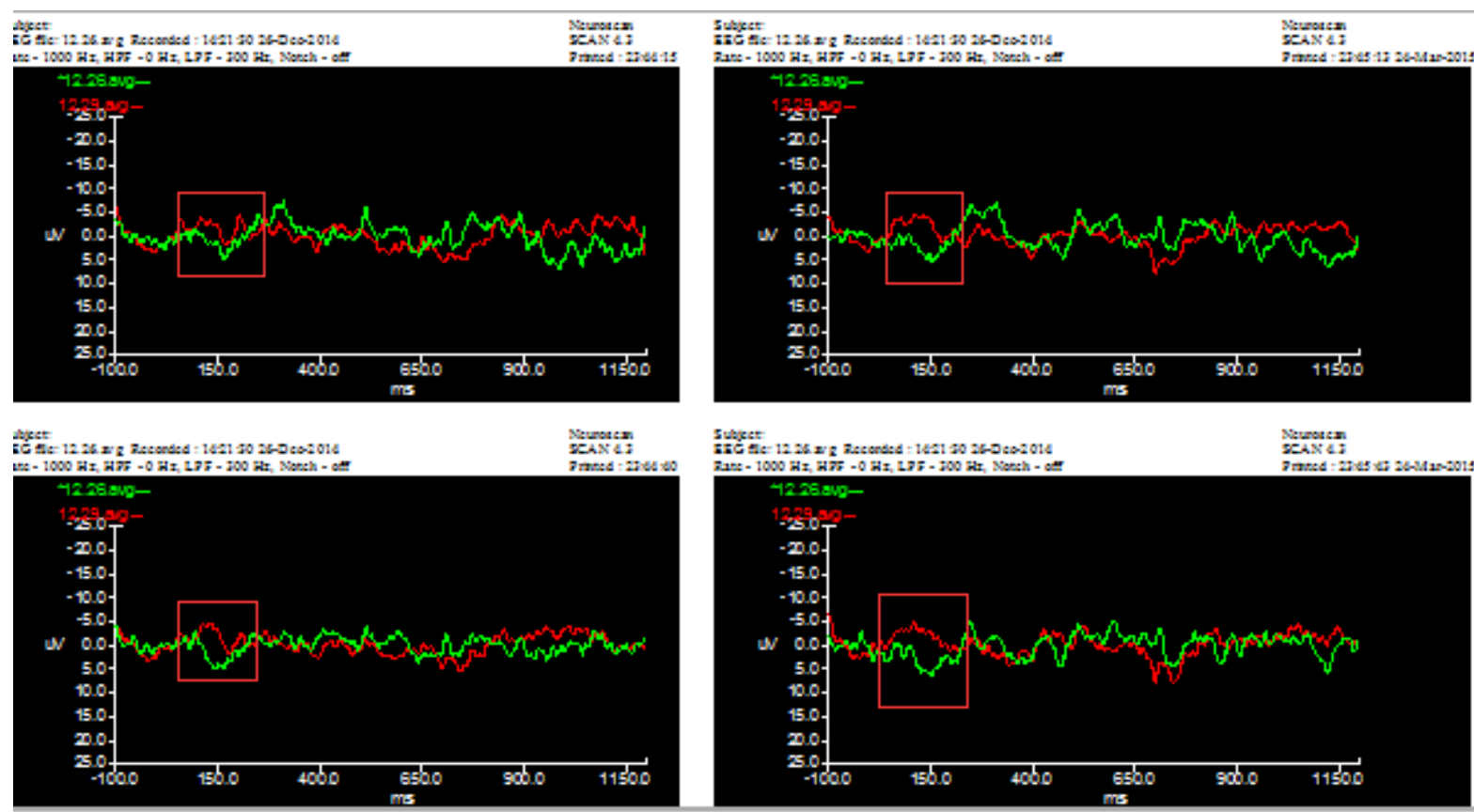

As you can see from Figure 1, the red line (dark line) that the subject is a reminder, light colored line indicates subjects did not receive any reminder, in different electrode, are reminders of the event, the results appear in the $1000 \mathrm{~ms}$ appeared in the thinking process of subjects (subjects EEG magnitude greater than non remind), it can be concluded that the different frequency components of EEG signals can reaction using different EEG game process.

In order to obtain the statistical results of multiple subjects, we use the Fisher distance for calculating.

The same experiments are conducted on 120 subjects, using the Fisher distance to calculate the EEG signals fewer than two modes of their choice of which Fisher distance between feature points, the larger, eventually and the following results can be obtained:

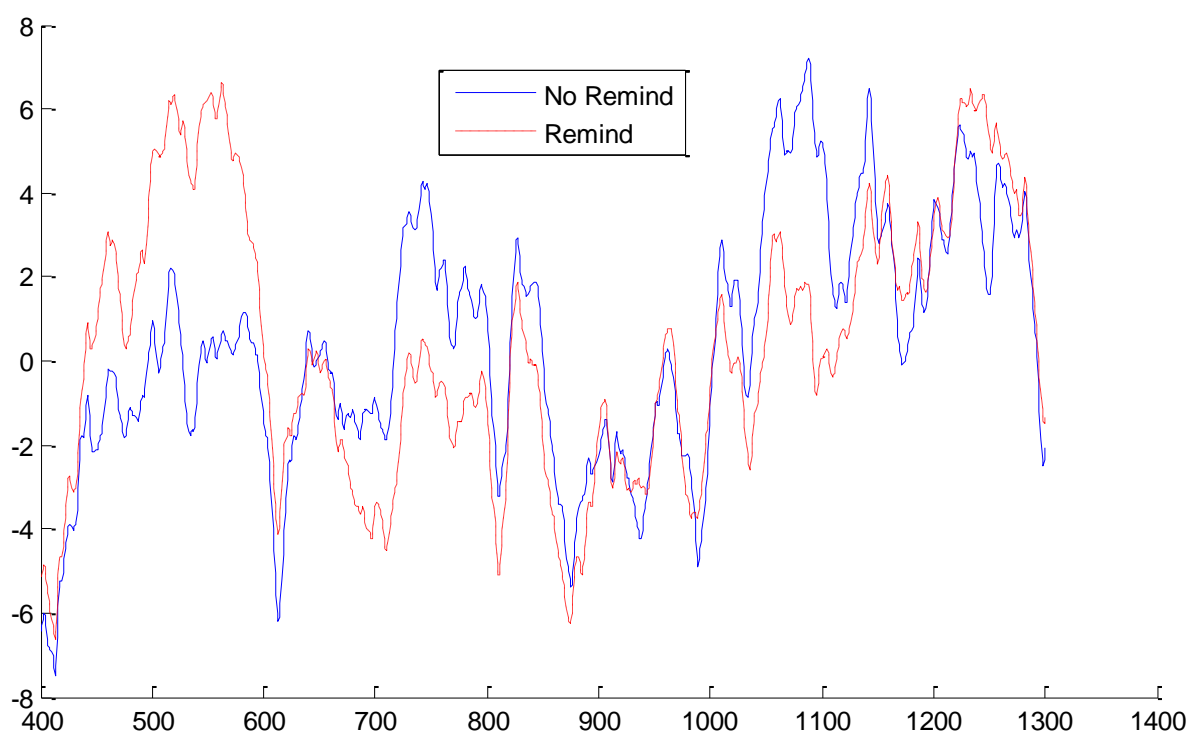




\section{Conclusion}

Study on the neural mechanism of decision, so far there are a few achievements, the reason is the limited acquisition method, this subject adopts double synchronous recording method, analysis in the decision making process of EEG signal specific features, and then back stepping generating neural mechanism of specific characteristics, which has important academic value to explain the neural mechanisms of fill modern theories of decision making.

\section{Acknowledgements}

This work was financially supported by IT projects of Jiangxi Office of Education [No. GJJ14765] and Nature of Jiangxi University of Technology [No. ZR13ZD05].

\section{References}

[1]Oberg S A K, Christie G J, Tata M S. Problem gamblers exhibit reward hypersensitivity in medial frontal cortex during gambling[J]. Neuropsychologia, 2011.

[2]Massar S A A, Rossi V, Schutter D, et al. Baseline EEG theta/beta ratio and punishment sensitivity as biomarkers for feedback-related negativity (FRN) and risk-taking[J]. Clinical Neurophysiology, 2012.

[3]Wölfling K, Mörsen C P, Duven E, et al. To gamble or not to gamble: At risk for craving and relapse-learned motivated attention in pathological gambling[J]. Biological psychology, 2011, 87(2): 275-281.

[4]Daeyeol Lee, Benjamin P.McGreevy, Dominic J.Barraclough, Learning and decision making in monkeys during a rock- paper- scissors game[J]. Cognitive Brain Research 2005, 25:416- 430. 